Pacific Journal of Mathematics

MANIFOLDS WITH POSITIVE CURVATURE 


\section{MANIFOLDS WITH POSITIVE CURVATURE}

\section{THEODORE FRANKEL}

o. Introduction and a conjecture. In $1936 \mathrm{~J}$. L. Synge [10] proved that an even dimensional orientable compact manifold $M_{n}$ with positive sectional curvature is simply connected. His proof was an application of a formula for the second variation of arc length derived by him in an earlier article. ${ }^{1}$ In the present paper we continue the study of positively curved manifolds again using the ideas of Synge and applying them to an only slightly different situation, namely to the "position" of certain submanifolds of $M$.

Theorem 1 states that two compact totally geodesic (see $\S 2$ for definitions) submanifolds $V_{r}$ and $W_{s}$ of $M_{n}$ must necessarily intersect if their dimension sum is at least that of $M$, i.e. if $r+s \geqq n$. As remarked above the proof is a straightforward continuation of Synge's method. Unfortunately totally geodesic submanifolds are not a too common occurrence.

If $M_{n}$ is a Kähler manifold ${ }^{2}$ the situation is much more satisfactory. There, instead of requiring $V$ and $W$ to be totally geodesic, we need only ask that they be complex analytic submanifolds (Theorem 2).

Examples of compact Riemannian manifolds of positive sectional curvature are the spheres, the real, complex and quaternionic projective spaces and the Cayley plane. Rauch [8] has shown that if the sectional curvatures do not differ too much from that of the sphere and if the space is simply connected, then it is itself topologically a sphere (see also the recent improvements by W. Klingenberg, Über kompakte Riemannsche Mannigfaltigkeiten, Math. Ann., 137 (1959), pp. 351-61). Berger [2] has shown that if $M_{n}$ is an even dimensional, simply connected manifold and if the sectional curvature $K$ satisfies $1 / 4 \leqq K \leqq 1$, then the manifold is one of the spaces listed above.

In the list the only Kähler manifolds are the complex projective $n$ spaces $P_{n}(\boldsymbol{C})$ with the usual Fubini metric. If $\left(e_{1}, e_{2}\right)$ is a pair of orthonormal tangent vectors to $P_{n}(\boldsymbol{C})$, then the sectional curvature $K\left(e_{1}, e_{1}\right)$ satisfies $1 / 4 \leqq K\left(e_{1}, e_{2}\right) \leqq 1$ with $K=1$ if and only if the plane $e_{1} \wedge e_{2}$ is a "complex direction." It may very well be that

ConjeCtuRE. The positively curved Kähler manifolds of complex dimension $n$ are analytically homeomorphic to $P_{n}(\boldsymbol{C})$. The Gauss Bonnet

Received January 11, 1960. Work supported in part by the National Science Foundation.

1 For completeness we include in $\S 1$ a derivation of the second variation formula.

2 Since the Ricci curvature of a positively curved manifold is positive, the Kähler manifold is a "Hodge manifold" and Kodaira's theorem [6] states that the manifold is algebraic. 
theorem shows that this is true for $n=1$. Using Theorem 2, A. Andreotti has shown that the conjecture is true for $n=2$ and his proof is presented in Theorem 3. It relies heavily on the known classification of algebraic surfaces. ${ }^{2}$

Difficulties in attempting to construct counter examples stem from the fact that the product of two positively curved manifolds has only nonnegative curvature (in the product metric). If $e_{1} \wedge e_{2}$ is a product plane (e.g., if $e_{1}$ is "horizontal" and $e_{2}$ is "vertical"), then $K\left(e_{1}, e_{2}\right)=0$ and this is the only time 0 curvature can occur. Our results in general do not apply to such spaces.

The last section is devoted to proving the existence of fixed points for certain maps, thus showing further similarities with $P_{n}(C)$.

I should like to thank A. Andreotti, E. Calabi and N. Hawley for discussions of the results.

1. Second variation of arc length. Our notation is as follows. $M_{n}$ is a complete $n$ dimensional Riemannian manifold and $V_{r}$ and $W_{s}$ are submanifolds of dimension $r$ and $s$ respectively. $\mathscr{C}(t)$ is a geodesic going from $\mathscr{C}(0)=P \in V$ to $\mathscr{C}(l)=Q \in W$ striking $V$ and $W$ orthogonally; $t$ represents arc length along $\mathscr{C} . X_{t}$ is a unit vector field that is displaced parallel along $\mathscr{C}$ and is tangent to $V$ and $W$ at $P$ and $Q$ respectively; $X_{t}$ (if it exists) is thus orthogonal to $\mathscr{C}$ for all $t$. Finally $T_{t}$ is the unit tangent vector to $\mathscr{C}$.

We construct a "variation" of the geodesic $\mathscr{C}$ as follows. We pass a small "ribbon" of surface through $\mathscr{C}$ that is tangent to $X_{t}$ at $\mathscr{C}(t)$. for all $t$ such that $0 \leqq t \leqq l$. This ribbon cuts $V$ and $W$ in two curves. We now pass curve segments on the ribbon tangent to $X_{t}$ at $\mathscr{C}(t)$, the curves varying smoothly from $V$ to $W$. The ribbon is chosen so "thin" that no two segments intersect. On each segment we use the directed arc length $\alpha$ from $\mathscr{C}$ as parameter and we may suppose that $-\varepsilon \leqq \alpha \leqq+\varepsilon$. Each point on the ribbon carries two coordinates $(t, \alpha)$ and we have two systems of coordinate curves $t=$ constant and $\alpha=$ constant (the original geodesic is of course $\alpha=0$ ). We have two coordinate vector fields $T=$ $\partial / \partial t$ and $X=\partial / \partial \alpha$ defined on the ribbon with $T=T_{t}$ at $(t, 0)$ and $X=X_{t}$ at this same point. The problem is to investigate the lengths of the curves $\alpha=$ constant.

We recall some facts and notation of Riemannian geometry (our notation follows [7]). We let $g(Y, Z)$ denote the Riemannian scalar product of two vectors $Y$ and $Z$; if $\left(x_{1}, \cdots, x_{n}\right)$ are local coordinates for $M$, then $g(Y, Z)=\sum_{i j} g_{i j} Y^{i} Z^{j}$. If $Y$ is a vector at a point and if $f$ is a function, then the covariant derivative of $f$ with respect to $Y$, written $\nabla_{Y}(f)$, is the directional derivative of $f$ in the direction $Y$. If $Z$ is a vector field, the covariant derivative of $Z$ with respect to $Y$ is again a 
vector, written $\nabla_{Y} Z$. If $Y$ is also a vector field, the Lie or commutator bracket of $Y$ and $Z$ is given by $[Y, Z]=Y Z-Z Y=\nabla_{Y} Z-\nabla_{Z} Y$. In particular, if $Y$ and $Z$ are coordinate vectors $\nabla_{Y} Z-\nabla_{Z} Y=[Y, Z]=0$. Hence in the case of our particular vectors we have

$$
\nabla_{X} T=\nabla_{T} X
$$

Next we have the Ricci operator identity

$$
\nabla_{Y} \nabla_{Z}-\nabla_{Z} \nabla_{Y}=R(Y, Z)+\nabla_{[Y, Z]}
$$

where $R(Y, Z)$ is, for each pair $(Y, Z)$, a linear transformation on tangent vectors. $R(Y, Z)$ is constructed from the Riemann curvature tensor and in terms of coordinates the transformation of vectors $U \rightarrow R(Y, Z) U$ is given by

$$
\sum_{i} U^{i} \frac{\partial}{\partial x^{i}} \rightarrow \sum_{i}\left(\sum_{j k l}-R_{j k l}^{i} Y^{k} Z^{i} U^{j}\right) \frac{\partial}{\partial x^{i}}
$$

$R(Y, Z)$ is skew symmetric; $R(Y, Z)=-R(Z, Y)$. In our case the Ricci identity becomes

$$
\nabla_{X} \nabla_{T}-\nabla_{T} \nabla_{X}=R(X, T) .
$$

The Riemannian sectional curvature corresponding to the 2-plane $T \wedge X$ is given by

$$
K(T, X)=g(R(X, T) T, X)=-g(R(X, T) X, T) .
$$

Finally we recall that the scalar product is "covariant constant," i.e.

$$
\frac{\partial}{\partial \alpha} g(Y, Z)=\nabla_{X} g(Y, Z)=g\left(\nabla_{X} Y, Z\right)+g\left(Y, \nabla_{X} Z\right) .
$$

The length of the curve $\alpha=$ constant is given by

$$
L(\alpha)=\int_{0}^{l} g(T, T)^{1 / 2} d t .
$$

LEMma ([9]). The first and second variations of arc length are

$$
\left\{\begin{array}{l}
L_{X}^{\prime}(0)=\left.\frac{d L}{d \alpha}\right|_{0}=0 \\
L_{X}^{\prime \prime}(0)=\left.\frac{d^{2} L}{d \alpha^{2}}\right|_{0}=g\left(\nabla_{X} X, T\right)_{Q}-g\left(\nabla_{X} X, T\right)_{P}-\int_{0}^{\imath} K(T, X) d t .
\end{array}\right.
$$

Proof.

$$
L^{\prime}(\alpha)=\int_{0}^{l} \frac{\partial}{\partial \alpha} g(T, T)^{1 / 2} d t=\int_{0}^{l} \nabla_{x} g(T, T)^{1 / 2} d t
$$


thus

$$
L^{\prime}(\alpha)=\int_{0}^{l} \frac{g\left(\nabla_{X} T, T\right)}{g(T, T)^{1 / 2}} d t
$$

But $g(T, T) \equiv 1$ along $\alpha=0$ ( $T$ is unit tangent to $\mathscr{C}(t)$ ) and so from (1) we get

$$
L^{\prime}(0)=\int_{0}^{\imath} g\left(\nabla_{X} T, T\right) d t=\int_{0}^{l} g\left(\nabla_{T} X, T\right) d t=0
$$

since $\nabla_{T} X=0$ for a parallel displaced $X$.

For the second variation we continue from (4)

$$
L^{\prime \prime}(\alpha)=\int_{0}^{l} \nabla_{X}\left\{\frac{g\left(\nabla_{T} X, T\right)}{g(T, T)^{1 / 2}}\right\} d t
$$

which expands to

$$
L^{\prime \prime}(0)=\int_{0}^{l} \nabla_{X} g\left(\nabla_{T} X, T\right) d t-\int_{0}^{\imath} g\left(\nabla_{T} X, T\right)^{2} d t .
$$

But $X$ is displaced parallel along $\mathscr{C} ; \nabla_{T} X=0$ and so the second integral vanishes. Thus

$$
L^{\prime \prime}(0)=\int_{0}^{l} g\left(\nabla_{X} \nabla_{T} X, T\right) d t+\int_{0}^{l} g\left(\nabla_{T} X, \nabla_{X} T\right) d t
$$

but again the second integral vanishes. Using (2) the first term becomes

$$
L^{\prime \prime}(0)=\int_{0}^{l} g\left(\nabla_{T} \nabla_{X} X, T\right) d t+\int_{0}^{l} g(R(X ; T) X, T) d t .
$$

The first integral transforms by means of

$$
g\left(\nabla_{T} \nabla_{X} X, T\right)=\nabla_{T} g\left(\nabla_{X} X, T\right)-g\left(\nabla_{X} X, \nabla_{T} T\right)=\frac{\partial}{\partial t} g\left(\nabla_{X} X, T\right)
$$

and using (3) we get the desired second variation.

The end terms in the second variation are interpreted as follows. $B_{T}(X)_{P} \equiv g\left(\nabla_{X} X, T\right)$ is the second fundamental form for $V$ at $P$ corresponding to the normal vector $T$, evaluated at the tangent vector $X$.

2. Real manifolds with positive curvature. A submanifold $V$ of a Riemannian $M_{n}$ is totally geodesic if any geodesic of $M$ that is tangent to $V$ at a point lies wholly in $V$. This implies that every geodesic of $V$ (in the naturally induced metric from $M$ ) is at the same time a geodesic of $M$. 
THEOREM 1. Let $M_{n}$ be a complete ${ }^{3}$ connected manifold with positive Riemannian sectional curvature and let $V_{r}$ and $W_{s}$ be compact totally geodesic submanifolds. If $r+s \geqq n$ then $V_{r}$ and $W_{s}$ have a non-empty intersection.

Proof. At first we assume that $V_{r}$ and $W_{s}$ are any compact submanifolds. We suppose they do not intersect. Then there is a shortest geodesic $\mathscr{C}(t)$, say of length $l>0$, from $V$ to $W$ and let $P$ and $Q$ be the points $\mathscr{C}(0)$ and $\mathscr{C}(l)$ respectively. Since $\mathscr{C}$ is the shortest geodesic from $V$ to $W$ it strikes $V$ and $W$ orthogonally. We will arrive at a contradiction by exhibiting a variation $X$ for which $L_{x}^{\prime \prime}(0)<0$, thus showing that $\mathscr{C}$ cannot be minimizing.

Let $\mathscr{V}_{0}^{-}$be the tangent space to $V_{r}$ at $P$. By parallel translation along $\mathscr{C}$ we get a subspace $\mathscr{V}_{i}^{\prime}$ of $\mathscr{C}$, the tangent space to $M_{n}$ at $Q$. Since $\mathscr{V}_{0}$ is orthogonal to $\mathscr{C}$ at $P, \mathscr{V}_{i}$ is also orthogonal to $\mathscr{C}$ at $Q$. Let $\mathscr{W}$ be the tangent space to $W_{s}$ at $Q$. Then $\mathscr{\mathscr { C }}$ and $\mathscr{W}$ are two subspaces of the linear space $\mathscr{C}$; moreover, both $\mathscr{V}_{l}$ and $\mathscr{W}$ are orthogonal to $\mathscr{C}$ at $Q$. Thus the dimension of their intersection is

$$
\operatorname{dim}\left(\mathscr{Y}_{\imath} \cap \mathscr{W}\right) \geqq r+s-(n-1) \geqq 1
$$

and thus $\mathscr{Y}_{l}$ and $\mathscr{W}$ have at least a one dimensional subspace in common. But this simply means that there is a unit vector $X_{0}$ tangent to $V$ at $P$ whose parallel translate is tangent to $W$ at $Q$. Let $X_{t}$ be the parallel translate of $X_{0}$ along $\mathscr{C}$. The term $-\int_{0}^{l} K(T, X) d t$ of the second variation formula is strictly negative by the curvature assumption.

So far $V$ and $W$ were arbitrary. To evaluate the end terms in the second variation we use the fact that $V$ and $W$ are totally geodesic. The variation vector $X_{t}$ is given. For the construction of the "ribbon" we can choose geodesics of $M$ through each $X_{t}$; since $X_{0}$ is tangent to $V$ at $P$ and since $V$ is totally geodesic, the geodesic through $X_{0}$ will lie entirely in $V$. Likewise the geodesic through $X_{l}$ will lie entirely in $W$. Thus the curves $\alpha=$ constant will have their endpoints on $V$ and $W$ as required for the variation. But since $X_{0}$ and $X_{l}$ are tangent vectors to geodesics of $M$ we have $\nabla_{X} X=0$ at $P$ and $Q$. Hence the end terms of the second variation formula vanish and we have

$$
L_{X}^{\prime \prime}(0)=-\int_{0}^{l} K(T, X) d t<0
$$

as desired.

We note that $g\left(\nabla_{X} X, T\right)_{P}=g\left(\nabla_{X} X, T\right)_{Q}=0$ is merely the statement that all second fundamental forms for a totally geodesic submanifold vanish identically.

${ }^{3}$ If the curvature is bounded away from $0, K \geqq \varepsilon>0$, the classical result of BonnetMyers states that $M_{n}$ is necessarily compact. 
There is at least one situation when totally geodesic submanifolds arise "naturally." If $f: M_{n} \rightarrow M_{n}$ is an isometric map of a Riemannian manifold into itself, then the set of fixed points $F=\{P \in M \mid f(P)=P\}$ has as components totally geodesic submanifolds (see [4]). Hence

CoRollary. If $f: M_{n} \rightarrow M_{n}$ is an isometry of a compact connected Riemannian manifold with positive curvature, then no two fixed set components can have dimension sum $\geqq n$.

3. Kähler manifolds with positive curvature. A Kähler manifold $M$ is a special type of Riemannian manifold whose underlying space is a complex manifold. There is a linear transformation $J$ on each tangent space that sends any vector $Y$ into a vector $J Y$ orthogonal to $Y(J$ represents multiplication by $\left.(-1)^{1 / 2}\right) . \quad J$ has the properties $J^{2}=-I$ and $g(J Y, J Z)=g(Y, Z)$ for all vectors $Y$ and $Z$ (this last property states that $g$ is a "Hermitian" metric). From $J$ we construct the Kähler exterior 2 -form $\omega$, defined by

$$
\omega(Y, Z)=g(J Y, Z) .
$$

$\omega$ is exterior because $\omega(Y, Z)=-\omega(Z, Y)$. All that has been said so far holds for any Hermitian manifold. The further condition defining a Kähler manifold can be stated as requiring that $\omega$ be covariant constant, $\nabla_{U} \omega=0$ for all vectors $U$; i.e., for any vector fields $Y$ and $Z$ we have

$$
\nabla_{U} \omega(Y, Z)=\omega\left(\nabla_{U} Y, Z\right)+\omega\left(Y, \nabla_{U} Z\right) .
$$

Since $g$ is also covariant constant we conclude that $J$ is also, i.e., we have the operator equation

$$
\nabla_{U}^{\circ} J=J \circ \nabla_{U}
$$

for any vector $U$.

A linear subspace $\mathscr{V}^{\prime}$ of the tangent space to a complex manifold at a point is said to be complex if it is invariant under $J, J: \mathscr{Y}^{\prime} \rightarrow \mathscr{Y}$. A submanifold is complex analytic if its tangent space at each point is complex.

When dealing with complex manifolds dimension subscripts will denote complex dimension.

The following result is easily true for $P_{n}(\boldsymbol{C})$ since it holds for the linear subspaces.

THEOREM 2. Let $M_{n}$ be a complete, connected Kähler manifold with positive sectional curvature and let $V_{r}$ and $W_{s}$ be compact complex analytic submanifolds. If $r+s \geqq n$, then $V_{r}$ and $W_{s}$ we have a nonempty intersection. 
Proof. The proof is again by contradiction, starting exactly as in Theorem 1. We again arrive at a variation vector $X_{t}$, parallel displaced along $\mathscr{C}$ and tangent to $V$ and $W$ at $P$ and $Q$ respectively. Now, however, we have additional information. Since $V$ and $W$ are complex analytic the vector field $J\left(X_{t}\right)$ is tangent to $V$ and $W$ at $P$ and $Q$ respectively. Further, from (6) we have $\nabla_{T} J\left(X_{t}\right)=J \nabla_{T} X_{t}=0$ since $X_{t}$ is parallel displaced. Thus $J\left(X_{t}\right)$ is also parallel displaced and gives us the same type of variation vector as $X_{t}$. We claim

the second variation corresponding to at least one of the fields $X_{t}$ or $J X_{t}$ is strictly negative

again giving a contradiction.

To prove our claim we suppose

$$
L_{X}^{\prime \prime}(0)=g\left(\nabla_{X} X, T\right)_{Q}-g\left(\nabla_{X} X, T\right)_{P}-\int_{0}^{l} K(T, X) d t \geqq 0 .
$$

By the hypothesis of positive curvature we conclude that

$$
g\left(\nabla_{X} X, T\right)_{Q}-g\left(\nabla_{X} X, T\right)_{P}>0 .
$$

We will be finished if we can show $g\left(\nabla_{J X} J X, T\right)_{Q}-g\left(\nabla_{J X} J X, T\right)_{P}<0$. But this is actually the case as follows from the fact that every second fundamental form of a complex analytic submanifold of a Kähler manifold is skew-hermitian, ${ }^{4}$ i.e.

$$
\begin{cases}g\left(\nabla_{J X} J X, T\right)_{P}=-g\left(\nabla_{X} X, T\right)_{P} & \text { for } \quad V \\ g\left(\nabla_{J X} J X, T\right)_{Q}=-g\left(\nabla_{X} X, T\right)_{Q} & \text { for } \quad W .\end{cases}
$$

The proof of this is simple and we include it here for completeness.

Let $\mathscr{R}$ be a complex analytic curve (real dimension 2 ) on $V$ tangent to $X_{0}$ and $J X_{0}$ at $P$. Then $X_{0}$ can be extended to a tangent vector field $X$ on $\mathscr{R}$ and of course $J X$ is an extension of $J X_{0}$. Since $X$ and $J X$ are tangent vector fields to $\mathscr{R}$ the commutator bracket $[J X, X]$ is again a vector field tangent to $\mathscr{R}$, and thus orthogonal to $T$ at $P$. Using $[J X, X]=\nabla_{J X} X-\nabla_{X} J X$ and $(6)$ and $J^{2}=-I$ we get at $P$

$$
\begin{aligned}
g\left(\nabla_{J X} J X, T\right) & =g\left(J \nabla_{J X} X, T\right)=g\left(J[J X, X]+J \nabla_{X} J X, T\right) \\
& =g(J[J X, X], T)-g\left(\nabla_{X} X, T\right) .
\end{aligned}
$$

Since $[J X, X]$ is tangent to $\mathscr{R}$, so is $J[J X, X]$ and so the first term vanishes and the result follows. Q.E.D.

${ }_{4}$ This is a reflection of the fact that Kähler submanifolds of a Kähler manifold are minimal submanifolds in the sense of the calculus of variations. Thus their mean curvatures vanish for all normal directions. 
4. Kähler surfaces with positive curvature. We now consider the case of Kähler surfaces $M_{2}$ (real dimension 4). We noticed previously ${ }^{2}$ that by Kodaira's theorem such a surface is necessarily algebraic.

We recall that an exceptional curve (of the first kind) arises in the following fashion. There is a surface $N_{2}$ and a point $P \in N_{2}$ such that $M_{2}$ is a quadratic transform [3] of $N_{2}$ and the exceptional curve is the quadratic transform of $p$. Thus exceptional curves result from blowing up a point $p$ of a surface by means of the Hopf $\sigma$-process; i.e., the point $p$ is replaced by the complex projective line $P_{1}(C)$ of complex directions at $p$. Since there clearly are curves that do not intersect the exceptional curve (hyperplane section of $N_{2}$ for example) we conclude from Theorem 2 that a positively curved compact Kähler surface has no exceptional curves (of the first kind).

THEOREM 3. A compact Kähler surface $M_{2}$ with positive sectional curvature is complex analytically homeomorphic to $P_{2}(\boldsymbol{C})$.

Proof (Andreotti). As mentioned before ${ }^{2}$ the Ricci curvature of a positively curved Kähler $M_{n}$ is positive. The negative of the exterior Ricci form represents the characteristic class of the canonical bundle $K$ over $M$. By Kodaira's "vanishing theorem" [5] we conclude $H^{p}\left(M_{n} ; \Omega^{0}\left(K^{1}\right)\right)=$ $0, p \neq n$, where $K^{i}$ is the line bundle $K \otimes \cdots \otimes K, i$ factors and where $\Omega^{0}\left(K^{i}\right)$ is the sheaf of germs of holomorphic sections of $K^{i}$. Thus the plurigenera $P_{i}=\operatorname{dim} H^{0}\left(M_{n} ; \Omega^{0}\left(K^{i}\right)\right)$ all vanish and since $M_{2}$ is simply connected the arithmetic genus $p_{a}=P_{1}-h^{1,0}=0$ also. We now apply results in the classification theory of surfaces, i.e., $n=2$. By a theorem of Castelnuovo-Enriques (for references see, for example, Zariski's book, Introduction to the problem of minimal models in the theory of algebraic surfaces, Math. Soc. of Japan, 1958, p. 84) we conclude that $M_{2}$ is rational. As we have just seen $M_{2}$ can have no exceptional curves (of the first kind). By a result of Andreotti [1] $M_{2}$ is either birationally equivalent, without exceptions, to $P_{2}(\boldsymbol{C})$ or else it is a ruled surface. Since the rulings would be compact curves that do not intersect, Theorem 2 eliminates this last possibility. Q.E.D.

5. Correspondences. A (holomorphic) correspondence of a complex manifold $N_{n}$ with itself is a complex analytic $n$ dimensional submanifold of $N_{n} \times N_{n}$.

A holomorphic map $f: N_{n} \rightarrow N_{n}$ gives rise to a correspondence, the graph $G(f)$ of $f ; G(f)=\left\{(p, f p) \mid p \in N_{n}\right\} . G(f)$ is of course a special type of correspondence since $f$ is single valued. Let $\Delta=\left\{(p, p) \mid p \in N_{n}\right\}$ be the diagonal of $N_{n} \times N_{n}$. It is clear that a map $f$ will have a fixed point whenever $G(f)$ intersects the diagonal $\Delta$. A correspondence will be said to have a fixed point if it intersects the diagonal. 
THEOREM 4. Every (holomorphic) correspondence of a connected compact Kähler manifold $N_{n}$ with positive curvature has a fixed point.

Proof. Again this is a simple known property of $P_{n}(\boldsymbol{C})$.

The correspondence is a complex analytic submanifold $V_{n}$ of $N_{n} \times N_{n}$. The same is true for the diagonal $\Delta$. We need only show that $V_{n}$ and $\Delta$ intersect, and this almost follows from Theorem 2. However, as pointed out in the introduction, $N_{n} \times N_{n}$ has only nonnegative curvature; product planes give 0 sectional curvature. This, however, is easily mended as follows.

In our previous notation $V_{n}=V, \Delta=W$ and $N_{n} \times N_{n}=M$. In the proof of Theorem 2 positive curvature occurs only in the statement $\int_{0}^{l} K(T, X) d t>0$. Now we can only say.

$$
\left\{\begin{array}{c}
L_{X}^{\prime \prime}(0)=\left(\nabla_{X} X, T\right)_{Q}-\left(\nabla_{X} X, T\right)_{P}-\int_{0}^{l} K(T, X) d t \\
\int K(T, X) d t \geqq 0
\end{array}\right.
$$

Again we suppose $L_{x}^{\prime \prime}(0) \geqq 0$.

Case 1. $\left(\nabla_{X} X, T\right)_{Q}-\left(\nabla_{X} X, T\right)_{P}>0$. Then from (8) we $L_{J X}^{\prime \prime}(0)<0$ and we are finished.

Case 2. $\left(\nabla_{X} X, T\right)_{Q}=\left(\nabla_{X} X, T\right)_{P}$ and $\int_{0}^{\imath} K(T, X) d t=0$. We will then be finished if we can show $\int_{0}^{l} K(T, J X) d t>0$. Now $\int_{0}^{l} K(T, X) d t=0$. means $T \wedge X$ is a product plane along $\mathscr{C}$, in particular at $Q \in W=\Delta$. Choose a real basis for the tangent space to $N_{n} \times N_{n}$ at $Q$ consisting of the $2 n$ "horizontal" orthonormal vectors $e_{1}, J e_{1}, \cdots, e_{n}, J e_{n}$ and the $2 n$ "vertical" orthonormal vectors $f_{1}, J f_{1}, \cdots, f_{n}, J f_{n}$. Since $T \wedge X$ is a. product plane the basis can be so chosen that

$$
\begin{aligned}
& X=(\cos \theta) e_{1}+(\sin \theta) f_{1} \\
& T=-(\sin \theta) e_{1}+(\cos \theta) f_{1} .
\end{aligned}
$$

Thus

$$
J X=(\cos \theta) J e_{1}+(\sin \theta) J f_{1} .
$$

This means that the only possibilities for $T \wedge J X$ to be a product plane are either $\cos \theta=0$ or $\sin \theta=0$, i.e., either $T= \pm e_{1}$ or $T= \pm f_{1}$. But $e_{1}$ and $f_{1}$ being respectively horizontal and vertical cannot be orthogonal to the diagonal $W=\Delta$ while the geodesic tangent $T$ must be. We thus conclude that if $T \wedge X$ is a product plane then $T \wedge J X$ cannot be. Hence $\int_{0}^{l} K(T, J X) d t>0$. Q.E.D. 
The isometries (rotations) of the 3-sphere without fixed points show that there is no real analogue of Theorem 4.

\section{REFERENCES}

1. A. Andreotti, On the complex structures of a class of simply connected manifolds, in the Lefschetz symposium volume Algebraic geometry and topology, Princeton, 1957.

2. M. Berger, Les variétés riemanniennes (1/4)-pincées. C. R. Acad. Sci. Paris, 250 (1960), $442-44$.

3. H. Hopf, Zur Topologie der komplexen Mannigfaltigkeiten, Studies and Essays presented to R. Courant, New York, (1948), 167-85.

4. S. Kobayashi, Fixed points of isometries, Nagoya Math. J., 13 (1958) 63-68.

5. K. Kodaira, On a differential-geometric method in the theory of analytic stacks, Proc. Nat. Acad. Sci. U. S. A., 39 (1953), 1268-73.

6. — On Kähler varieties of restricted type, Ann. of Math., 60 (1954), 28-48.

7. K. Nomizu, Lie groups and differential geometry, Math, Soc. Japan (1956).

8. H. E. Rauch, A contribution to differential geometry in the large, Ann. of Math., 54 (1951), 38-55.

9. J. L. Synge, The first and second variations of length in Riemannian space, Proc. London Math. Soc., 25 (1926).

10. — On the connectivity of spaces of positive curvature, Quart. J. Math. (Oxford series) 7 (1936), 316-20.

STANFORD UNIVERSITY 


\section{PACIFIC JOURNAL OF MATHEMATICS}

\section{EDITORS}

Ralph S. Phillips

Stanford University

Stanford, California

F. H. BrownelL

University of Washington

Seattle 5 , Washington
A. L. Whiteman

University of Southern California Los Angeles 7. California

L. J. PAIGE

University of California

Los Angeles 24, California

ASSOCIATE EDITORS
E. F. BECKENBACH
D. DERRY
H. L. ROYDEN
E. G. STRAUS
T. M. CHERRY
M. OHTSUKA
E. SPANIER
F. WOLF

\section{SUPPORTING INSTITUTIONS}

UNIVERSITY OF BRITISH COLUMBIA

CALIFORNIA INSTITUTE OF TECHNOLOGY

UNIVERSITY OF CALIFORNIA

MONTANA STATE UNIVERSITY

UNIVERSITY OF NEVADA

NEW MEXICO STATE UNIVERSITY

OREGON STATE COLLEGE

UNIVERSITY OF OREGON

OSAKA UNIVERSITY

UNIVERSITY OF SOUTHERN CALIFORNIA
STANFORD UNIVERSITY

UNIVERSITY OF TOKYO

UNIVERSITY OF UTAH

WASHINGTON STATE COLLEGE,

UNIVERSITY OF WASHINGTON

AMERICAN MATHEMATICAL SOCIETY

CALIFORNIA RESEARCH CORPORATION

HUGHES AIRCRAFT COMPANY

SPACE TECHNOLOGY LABORATORIES

NAVAL ORDNANCE TEST STATION

Printed in Japan by International Academic Printing Co., Ltd., Tokyo, Japan

Reprinted 1966 in the United States of America 


\section{Pacific Journal of Mathematics}

\section{Vol. 11, No. $1 \quad$ November, 1961}

A. A. Albert, Generalized twisted fields ............................ 1

Richard Arens, Operational calculus of linear relations ................... 9

John Herbert Barrett, Disconjugacy of a self-adjoint differential equation of the fourth order ....................................... 25

Paul Richard Beesack, Hardy's inequality and its extensions ............... 39

Julius Rubin Blum and David Lee Hanson, On invariant probability measures.

II .............................................

Robert Allen Bonic, Symmetry in group algebras of discrete groups.......... 73

R. Creighton Buck, Multiplication operators ...................... 95

Jack Gary Ceder, Some generalizations of metric spaces ................. 105

Meyer Dwass, Random crossings of cumulative distribution functions ......... 127

Albert Edrei, Wolfgang H. J. Fuchs and Simon Hellerstein, Radial distribution and

deficiencies of the values of a meromorphic function ............... 135

William Cassidy Fox, Harmonic functions with arbitrary local singularities ..... 153

Theodore Thomas Frankel, Manifolds with positive curvature ............... 165

Avner Friedman, A strong maximum principle for weakly subparabolic

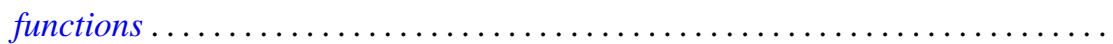

Watson Bryan Fulks and J. O. Sather, Asymptotics. II. Laplace's method for

multiple integrals ......................................

Adriano Mario Garsia and Eugene Richard Rodemich, An embedding of Riemann

surfaces of genus one ..................................... 193

Irving Leonard Glicksberg, Weak compactness and separate continuity......... 205

Branko Grünbaum, On a conjecture of H. Hadwiger .................. 215

Frank J. Hahn, On the action of a locally compact group on $E_{n} \ldots \ldots \ldots \ldots \ldots . . \ldots 221$

Magnus R. Hestenes, Relative hermitian matrices ..................... 225

G. K. Kalisch, On similarity invariants of certain operators in $L_{p} \ldots \ldots \ldots \ldots .247$

Yitzhak Katznelson and Walter Rudin, The Stone-Weierstrass property in Banach

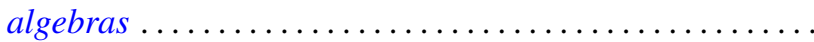

Samir A. Khabbaz, The subgroups of a divisible group $G$ which can be represented as intersections of divisible subgroups of $G \ldots \ldots \ldots \ldots \ldots \ldots \ldots \ldots \ldots . \ldots \ldots 7$

Marvin Isadore Knopp, Construction of a class of modular functions and forms .......................................... 275

Charles Alan McCarthy, Commuting Boolean algebras of projections .......... 295

T. M. MacRobert, Transformations of series of E-functions ................ 309

Heinz Renggli, An inequality for logarithmic capacities ................. 313

M. S. Robertson, Applications of the subordination principle to univalent functions .......................................... 315

David Sachs, Partition and modulated lattices ..................... 325

Frank S. Scalora, Abstract martingale convergence theorems ............... 347

Elbert A. Walker, Torsion endomorphic images of mixed Abelian groups ........ 375

Morgan Ward, The prime divisors of Fibonacci numbers................. 379

Charles R. B. Wright, On the nilpotency class of a group of exponent four....... 387 